\title{
QoS Management in WSN-MCN Convergence Network Using Priority Based Traffic Models
}

\author{
Anita Swain, Arun Kumar Ray \\ Kalinga Institute of Industrial Technology, (Deemed to be University), Bhubaneswar, India
}

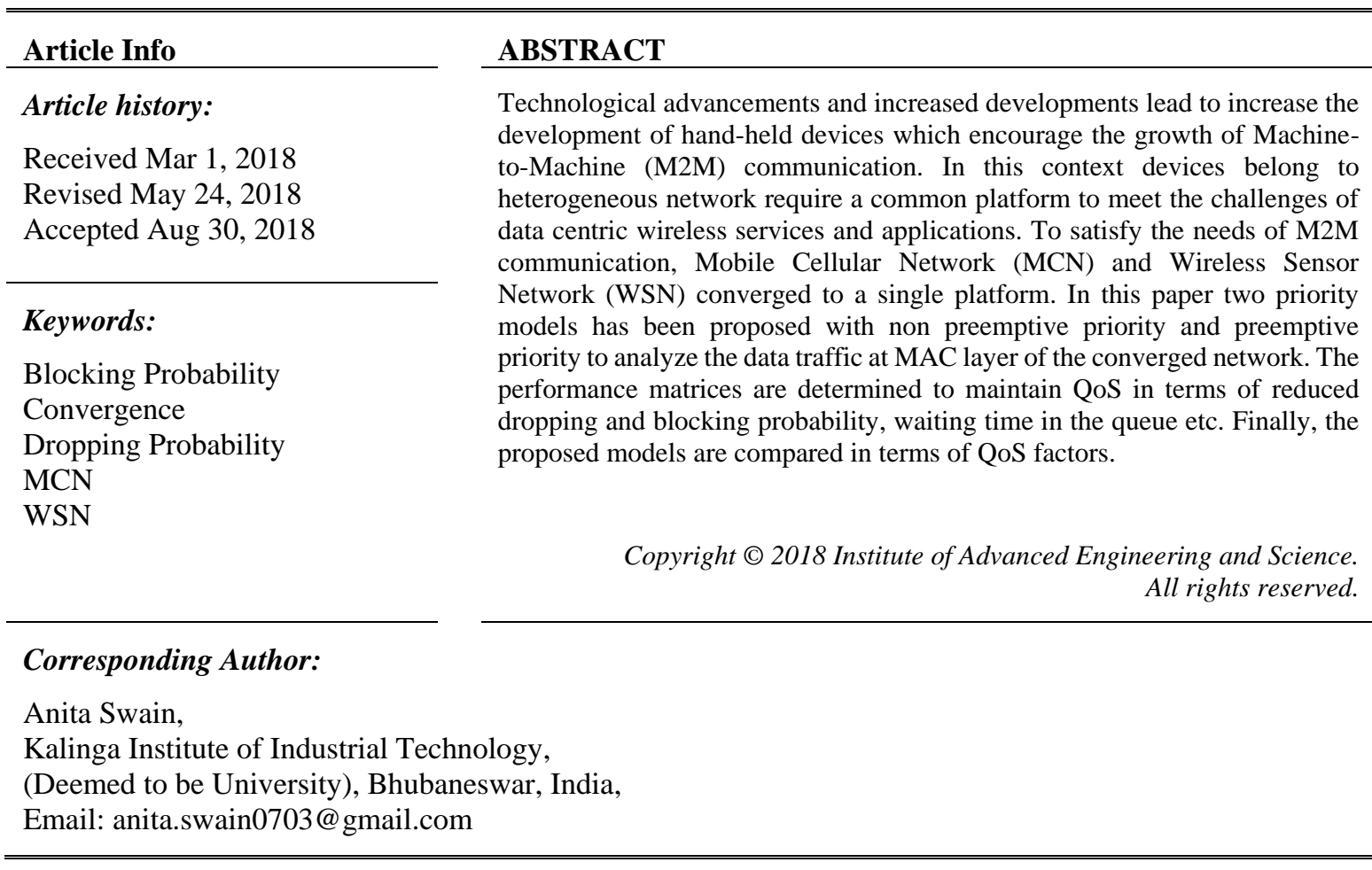

\section{INTRODUCTION}

Networking technologies allow a large number of diversity of devices such as mobile phones, laptops, TVs, personal computers, speakers, lights and electronic appliances to be connected in a seamless manner. This paradigm creates a possibility of machine-to-machine (M2M) communication and exchange of information. M2M communications is characterized by low power, low cost, and less human involvement [1, 2]. At this point, the WSN is going to satisfy the characteristics of M2M communication using static and mobile sensor nodes. However the deploying strategy of WSN depends upon the communication standard. Zigbee is one of the most appropriate communication standard for WSN [3]. However the mobile sensor nodes are more flexible to real time applications than the static sensor nodes $[4,5]$. In an integrated network of MCN and WSN, the front sensing part is WSNs which can be flexibly deployed to detect different types of sensory data and the $\mathrm{MCN}$ act as a background network for data transmission. These dynamic behavior of WSN encourages to create a platform for WSN and MCN to converge and establish a common network. Because MCN has the advantages of large coverage and powerful user terminals, WSN and MCN convergence is indispensable for supporting M2M communications [6]. The convergence of WCN and MSN can also benefit each other: (I) For WSN, the MCN can provide optimization to prolong WSN life time, provide quality of service (QoS) and improve syetem performance; (II) For MCN, WSN can extend the intelligent application range of MCN, i.e. WSN can provide real-time measurement results to MCN users. M2M communication is based on ubiquitous technology of WSN and MCN, which uses the cellular system as the backbone of the network. The two heterogeneous networks WSN and MCN, come together for the convergence to support the data centric services and application of M2M communication.

Convergence of WSN and MCN is an emerging research field in wireless communication [7]. The literature explores few researches on the issues of WSN and MCN convergence network with its 
application oriented implementation [8-11]. The idea of converging WSN and MCN and its comparison with the integrated network of WSN and MCN is given in [12]. However the integration of WSN and MCN has been already implemented through the gateway. In the integrated WSN and MCN, the architecture is hierarchical and the gateway is just a data channel to exchange information between the two independent protocol stacks. In WSN and MCN convergence network the gateway plays an important role for the convergence of two networks. Therefore, gateway selection in the convergence network is coming out as a research issues which need to be addressed [13]. The optimal gateway selection mechanism selects the most appropriate gateway for integration of WSN and MCN $[14,15]$.

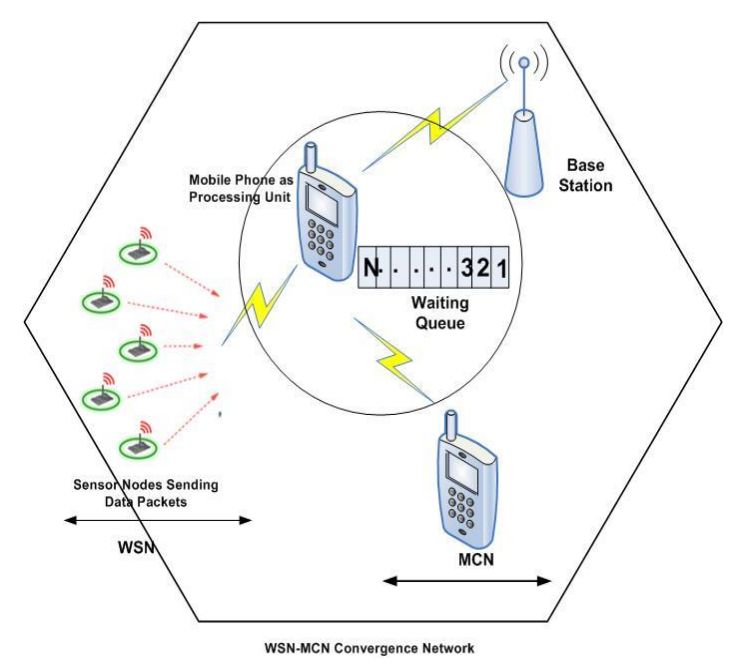

Figure 1. Data flow in a WSN-MCN convergence network

The flat architecture of convergence network as compared to hierarchical of integrated WSN and MCN network is going to be helpful for higher research [6]. The advantages of the flat architecture includes the sensor nodes to overhear the direct control signalling from the base station of MCN where as MCN can directly control the efficiency of WSN. For real convergence, the data channels between two protocols stacks need to be implemented for information exchange. For smooth exchange of control signalling some crossMAC should be designed. Considering the heavy traffic generated from WSN as well as MCN in convergence network, the MAC layer of convergence network requires new resource allocation scheme to achieve the Quality of Service (QoS). A cellular assisted Quality of Service (QoS) resource allocation algorithm has been proposed to reduce collision by taking the mobile terminal as gateway which acts as service scheduler for high priority service [16-19].

WSN generates a huge amount of data on timely basis which need to be transmitted by the gateway MS to the base station. But in the convergence network MCN component is mainly responsible for realtime data transmission for voice calls and videos rather than the data transmission in WSN component. So to handle the data traffic in convergence network there is a need for data traffic controlling process to maintain the QoS for MCN as well as WSN.

In this paper, we have focused on the data flow in the MAC layer of WSN and MCN convergence network for QoS management [20]. We have considered preemptive and non preemptive queuing model by giving emphasis on the priority of data packet to enter in to the queue. We have proposed a priority and a non priority based queuing model for WSN and MCN convergence network where in case of priority, MCN is taken as higher and WSN is of lower priority as shown in Figure 1. QoS parameters in MAC layer such as probability of dropping data packets, probability of data packet in services of WSN and MCN has been analyzed mathematically with numerical results.

The rest of the paper is organized as follows: Section 2 describes the proposed system model for priority and non priority services. In Section 3, we studied the performance factors of MCN and WSN convergence. Finally, we conclude our work in Section 4. 


\section{SYSTEM MODEL DESCRIPTION}

We consider a two priority system model for WSN-MCN Convergence network, where MCN data packets considered being the highest priority and WSN data packets considered to be the lowest priority for service at mobile system (MS). The packets arrival for both MCN and WSN follow a Poisson process at a rate $\lambda_{\mathrm{h}}$ and $\lambda_{\mathrm{l}}$ respectively. Data packets of both MCN and WSN get served by the mobile station of mobile cellular network and the service time is an exponentially distributed random variable with mean $1 / \mu_{\mathrm{h}}$ and $1 / \mu_{1}$ respectively. The data traffic of both the networks offered to the queue if the service point is considered to be busy. The traffic load is denoted by $\rho_{\mathrm{i}}=\lambda_{\mathrm{i}} / \mu_{\mathrm{i}}$, where $\mathrm{i}=\mathrm{h}$ or 1 , depends on MCN or WSN data service respectively. A mobile device is assumed to be equipped with a memory size of $\mathrm{N}$ (buffer space having $\mathrm{N}=1,2 \ldots \mathrm{N}$ ) to hold incoming data packets in a queue (both from MCN and WSN) till they get processed. When the queue size is equal to $\mathrm{N}$, the incoming data packets are dropped.

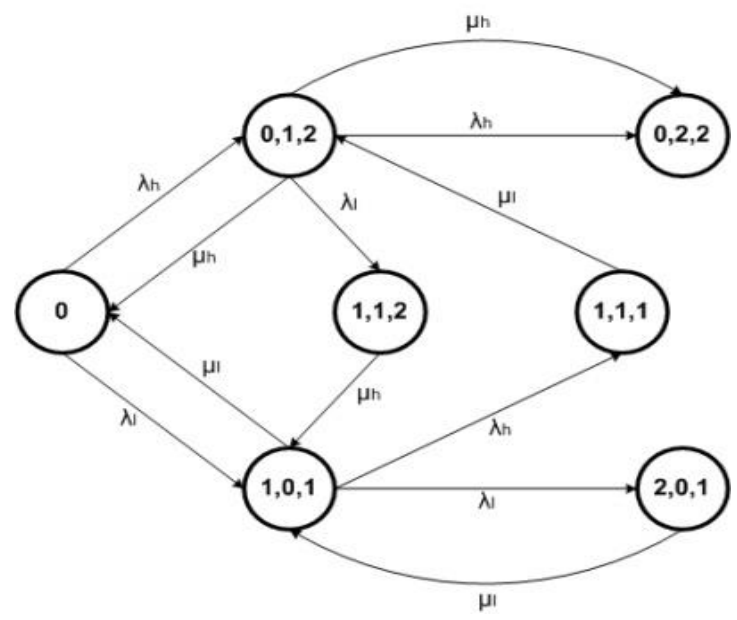

Figure 2. State transition diagram for non-preemptive priority system

In this paper QoS measurement is done by analyzing the arrival mechanism, service mechanism and buffering mechanism to reduce data dropping and waiting time in the data queue [21]. Here we have proposed a non-preemptive priority and preemptive priority model with 2-Priority finite buffer queuing system. The results of both the models are compared and discussed in numerical section.

\subsection{Analysis of Non-Preemptive Priority Model}

In this model we assume a non-preemptive priority system, where the ongoing service of WSN data is allowed to complete its service even if a high priority MCN data arrives to the mobile device for service. The MCN data admits into the queue and wait until the service point is free. Here we consider a two priority system with buffer capacity $\mathrm{N}=2$. State transition diagram for the above model using Markov chain is shown in Figure 2. The steady state probabilities are derived using balanced equations.

Let $\mathrm{P} i, \mathrm{j}, \mathrm{k}$ be the state probabilities where $\mathrm{i}(\mathrm{i}=0,1,2, \ldots)$ denotes the state of low priority WSN data, $\mathrm{j}(\mathrm{j}=0,1,2, \ldots)$ denotes the state of high priority MCN data and $\mathrm{k}$ denotes the current service status of data i.e. MCN or WSN data. The value of $\mathrm{k}=1$, denotes WSN data and $\mathrm{k}=2$, denotes MCN data. The algorithm for data flow in Non Preemptive priority model is presented in algorithm 1. In this algorithm we have initialized the system at initial state when time $\mathrm{t}=0$ and the mobile station is free. The buffer has empty space to admit requesting $\mathrm{MCN}$ or WSN service. When a new request is admitted to queue the current status of queue is increased by one. In case the buffer is full the network request is dropped.

Using probabilistic argument we obtain the following balanced equations:

$$
\begin{aligned}
& P_{(0)}\left(\lambda_{h}+\lambda_{l}\right)=P_{(0,1,2)} \mu_{h}+P_{(1,0,1)} \mu_{l} \\
& P_{(0,1,2)}\left(\lambda_{l}+\lambda_{h}+\mu_{h}\right)=P_{(0,2,2)} \mu_{h}+P_{(1,1,1)} \mu_{l}+P_{(0)} \lambda_{h} \\
& P_{(1,0,1)}\left(\lambda_{l}+\lambda_{h}+\mu_{l}\right)=P_{(0)} \lambda_{l}+P_{(1,1,2)} \mu_{h}+P_{(2,0,1)} \mu_{l}
\end{aligned}
$$




$$
\begin{aligned}
& P_{(0,2,2)} \mu_{h}=P_{(0,1,2)} \lambda_{h} \\
& P_{(1,1,2)} \mu_{h}=P_{(0,1,2)} \lambda_{l} \\
& P_{(1,1,1)} \mu_{l}=P_{(1,0,1)} \lambda_{h} \\
& P_{(2,0,1)} \mu_{l}=P_{(1,0,1)} \lambda_{l}
\end{aligned}
$$

Solving for individual state probabilities, let us assume:

$$
\begin{aligned}
& P_{(2,0,1)}=k \\
& P_{(1,0,1)}=\frac{k \mu_{l}}{\lambda_{l}} \\
& P_{(1,1,1)}=\frac{k \lambda_{h}}{\lambda_{l}} \\
& P_{(0)}=\frac{k \mu_{l}\left(\lambda_{h} \mu_{h}+\lambda_{l} \mu_{l}+\mu_{l} \mu_{h}\right)}{\lambda_{l}^{2}\left(\lambda_{l}+\lambda_{h}+\mu_{h}\right)} \\
& P_{(0,1,2)}=\frac{k \mu_{l} \lambda_{h}\left(\lambda_{l}+\lambda_{h}+\mu_{l}\right)}{\lambda_{l}^{2}\left(\lambda_{l}+\lambda_{h}+\mu_{h}\right)} \\
& P_{(0,2,2)}=\frac{k \mu_{l} \lambda_{h}^{2}\left(\lambda_{l}+\lambda_{h}+\mu_{l}\right)}{\lambda_{l}^{2} \mu_{h}\left(\lambda_{l}+\lambda_{h}+\mu_{h}\right)} \\
& P_{(1,1,2)}=\frac{k \mu_{l} \lambda_{h}\left(\lambda_{l}+\lambda_{h}+\mu_{l}\right)}{\lambda_{l} \mu_{h}\left(\lambda_{l}+\lambda_{h}+\mu_{h}\right)}
\end{aligned}
$$

Using normalization condition for the equation as:

$$
P_{(0)}+P_{(0,1,2)}+P_{(1,0,1)}+P_{(0,2,2)}+P_{(1,1,2)}+P_{(1,1,1)}+P_{(2,0,1)}=1
$$

Where $\mathrm{k}=1+\frac{\lambda_{h}}{\lambda_{l}}+\frac{\mu_{l}}{\lambda_{l}}+\frac{\mu_{l} \lambda_{h} r}{\lambda_{l} \mu_{h}}+\frac{\mu_{l} \lambda_{h}{ }^{2} r}{\lambda_{l}{ }^{2} \mu_{h}}+\frac{\mu_{l} \lambda_{h} r}{\lambda_{l}{ }^{2}}+\frac{\mu_{l}\left(\lambda_{h} \mu_{h}+\lambda_{l} \mu_{l}+\mu_{l} \mu_{h}\right)}{\lambda_{l}{ }^{2}\left(\lambda_{l}+\lambda_{h}+\mu_{h}\right)}$

And $\mathrm{r}=\frac{\lambda_{l}+\lambda_{h}+\mu_{l}}{\lambda_{l}+\lambda_{h}+\mu_{h}}$

Algorithm 1 Algorithm for Mobile Station (MS) Allocation Using Non Preemptive priority Model

MS (t) is the initial State of the MS at Time $t=0$ and Queue Size $<\mathrm{N}$

If MCN Data Service request then

Allocate to MS for Service;

MS is Busy;

Else

If MS is Busy AND MCN Request OR WSN Request then

Queue the Request;

$\mathrm{N}=\mathrm{N}+1$;

Else

Drop the Request;

End if

End if 
If WSN Data Service request then

Allocate to MS for Service;

MS is Busy;

Else

If MS is Busy AND WCN Request OR MSN Request then

Queue the Request;

$\mathrm{N}=\mathrm{N}+1$;

Else

End if

Drop the Request;

End if

\subsection{Performance Indices (Non-Preemptive Priority Model)}

In this section various performance matrices calculated to maintain QoS of the system. The probability that arriving data packets from MCN and WSN is dropped due to busy mobile station and buffer space in queue is full is denoted as $\mathrm{P}_{\mathrm{D}}$ (non-preemptive):

$\mathrm{P}_{\mathrm{D}}($ non-preemptive $)=\mathrm{P}_{(0,2,2)}+\mathrm{P}_{(1,1,1)}+\mathrm{P}_{(2,0,1)}+\mathrm{P}_{(1,1,2)}$.

The probability that mobile station is busy in serving low priority WSN data packets without preemption is:

Pwsn $=\mathrm{P}(1,0,1)+\mathrm{P}(2,0,1)+\mathrm{P}(1,1,1)$.

The probability that mobile station is busy in serving at least one MCN data packet is:

Pmcn $=\mathrm{P}(0,1,2)+\mathrm{P}(0,2,2)+\mathrm{P}(1,1,2)$.

When the mobile device is busy, arriving MCN and WSN data packets has to wait in the queue. The waiting time in queue is denoted as Wq (non-Preemptive) and can be expressed as

$$
\mathrm{Wq}_{(\text {non }- \text { Preemptive) }}=\mathrm{P}_{(0,2,2)}+\mathrm{P}_{(1,1,1)}+\mathrm{P}_{(2,0,1)}+\mathrm{P}_{(1,1,2)} \text {. }
$$

\subsection{Analysis of Preemptive Priority Model}

In WSN-MCN convergence network, MCN data packets have its priority over WSN data packets because of its real time application. Hence, WSN data packets can be delayed by passing the MCN data on a priority basis. Hence a preemptive priority model has been introduced to handle the preemption of WSN packets which are currently in service, upon arrival of MCN data. In this model the service time is exponentially distributed and it will satisfy memory less property, hence the results will be same for preemptive resume and preemptive non resume case. In this model both MCN and WSN data are admitted. If MCN data is in service and WSN data requests for service then it will wait in queue till mobile station gets free. And if the queue is full, then the requesting WSN data are dropped. On the other hand if MCN data arrives for service, it preempts the ongoing service of WSN data which will lose from the process.

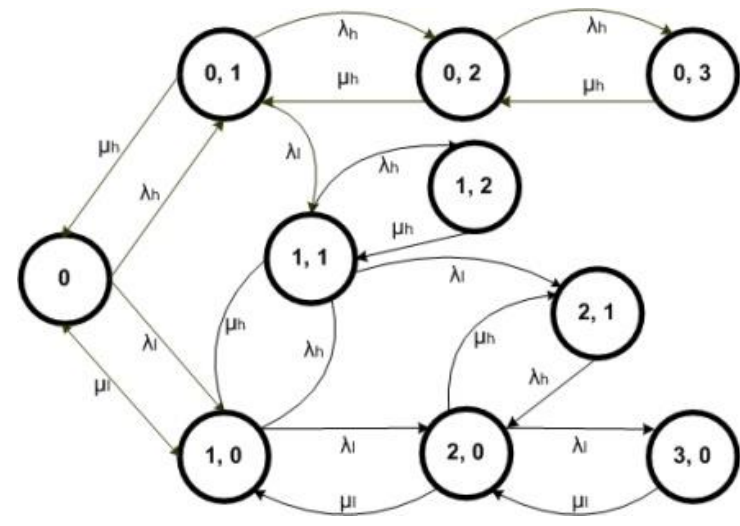

Figure 3. State transition diagram for preemptive priority system 
We consider a two priority system with buffer capacity $\mathrm{N}$ is equal to 2 . The state transition diagram for the above model using Markov chain is shown in Figure 3. Individual state probabilities can be represented as $\mathrm{P} i, j$ where $\mathrm{i}(\mathrm{i}=0,1,2, \ldots)$ denotes the state of low priority WSN data and $\mathrm{j}(\mathrm{j}=0,1,2, \ldots)$ denotes the state of high priority MCN data. The algorithm for data flow in Preemptive priority model is presented in algorithm 2. This algorithm discussed the system is at initial state when time $\mathrm{t}=0$ and the mobile station is free. The system considers priority method with preemption for MCN and WSN service request.

For WSN no preemption is permitted but for MCN the ongoing service of WSN is preempted from the system. The buffer has empty space to admit requesting MCN or WSN service. When a new request is admitted to the queue the current status of queue is increased by one. In case the buffer is full the network request is dropped.

Using probabilistic argument the steady state balanced equations are as follows:

$$
\begin{aligned}
& P_{(0)}\left(\lambda_{l}+\lambda_{h}\right)=P_{(0,1)} \mu_{h}+P_{(1,0)} \mu_{l} \\
& P_{(0,1)}\left(\lambda_{l}+\lambda_{h}+\mu_{h}\right)=P_{(0,2)} \mu_{h}+P_{(0)} \lambda_{h} \\
& P_{(1,0)}\left(\lambda_{l}+\lambda_{h}+\mu_{l}\right)=P_{(2,0)} \mu_{l}+P_{(1,1)} \mu_{h}+P_{(0)} \lambda_{l} \\
& P_{(1,1)}\left(\lambda_{l}+\lambda_{h}+\mu_{h}\right)=P_{(1,0)} \lambda_{h}+P_{(1,2)} \mu_{h}+P_{(0,1)} \lambda_{l} \\
& P_{(1,2)} \mu_{h}=P_{(1,1)} \lambda_{h}+P_{(0,2)} \lambda_{l} \\
& P_{(2,1)} \mu_{h}=P_{(1,1)} \lambda_{l}+P_{(2,0)} \lambda_{h} \\
& P_{(0,2)}\left(\lambda_{l}+\mu_{h}\right)=P_{(0,1)} \lambda_{h} \\
& P_{(2,0)}\left(\lambda_{h}+\mu_{l}\right)=P_{(1,0)} \lambda_{l}+P_{(2,1)} \mu_{h}
\end{aligned}
$$

Solving for individual state probabilities, let us assume,

$$
\begin{aligned}
& P_{(0,2)}=k \\
& P_{(0)}=\frac{k\left(\lambda_{l}+\mu_{h}\right)^{2}+\lambda_{l}^{2} \lambda_{h}^{2}}{\lambda_{h}^{2}} \\
& P_{(0,1)}=\frac{k\left(\lambda_{l}+\mu_{h)}\right.}{\lambda_{h}} \\
& P_{(1,0)}=\frac{k\left(M\left(\lambda_{l}+\lambda_{h}\right) \lambda_{h}-\mu_{h}\left(\lambda_{l}+\mu_{h}\right)\right)}{\mu_{l} \lambda_{h}} \\
& P_{(1,1)}=k L \\
& P_{(2,0)}=\frac{k \lambda_{l}\left(M\left(\lambda_{l}+\lambda_{h}\right) \lambda_{h}-\mu_{h}\left(\lambda_{l}+\mu_{h}\right)+\mathrm{L} \mu_{l} \lambda_{h}\right.}{\mu_{l}^{2} \lambda_{h}} \\
& P_{(1,2)}=\frac{k\left(\lambda_{l}+\mathrm{L} \lambda_{h}\right)}{\mu_{h}} \\
& P_{(2,1)}=\frac{k \lambda_{l}}{\mu_{h}}\left[\frac{\lambda_{h}}{\mu_{l}}\left(\frac{M\left(\lambda_{l}+\lambda_{h}\right) \lambda_{h}-\mu_{h}\left(\lambda_{l}+\mu_{h}\right)}{\mu_{l} \lambda_{h}}+L\right)+L\right]
\end{aligned}
$$




$$
\begin{aligned}
& P_{(0,3)}=\frac{k \lambda_{h}}{\mu_{h}} \\
& P_{(3,0)}=\frac{k \lambda_{l}^{2}}{\mu_{l}^{2}}\left[\frac{M\left(\lambda_{l}+\lambda_{h}\right) \lambda_{h}-\mu_{h}\left(\lambda_{l}+\mu_{h}\right)}{\mu_{l} \lambda_{h}}+L\right]
\end{aligned}
$$

Using normalization condition for the above equations as:

$$
P_{(0)}+P_{(0,1)}+P_{(1,0)}+P_{(1,1)}+P_{(0,2)}+P_{(2,0)}+P_{(1,2)}+P_{(2,1)}+P_{(0,3)}+P_{(3,0)}=1
$$

Where,

$$
\begin{gathered}
\mathrm{M}=\frac{\left(\lambda_{l}+\mu_{h}\right)^{2}+\lambda_{l}{ }^{2} \lambda_{h}{ }^{2}}{\lambda_{h}{ }^{2}} \text { and } \mathrm{L}=\frac{\lambda_{l}}{\lambda_{h}}+\frac{\lambda_{l}}{\left(\lambda_{l}+\mu_{h}\right)}+\frac{M\left(\lambda_{l}+\lambda_{h}\right) \lambda_{h}}{\mu_{l}\left(\lambda_{l}+\mu_{h}\right)}-\frac{\mu_{h}}{\mu_{l}} \\
\mathrm{~K}= \\
\left.M+\frac{\left(\lambda_{l}+\mu_{h}\right)}{\lambda_{h}}+\left(\frac{1}{\mu_{l} \lambda_{h}}\right) M \lambda_{h}\left(\lambda_{l}+\lambda_{h}\right)-\mu_{h}\left(\lambda_{l}+\mu_{h}\right)+1+1+\frac{\lambda_{l}}{\mu_{l}} \frac{M\left(\lambda_{l}+\lambda_{h}\right) \lambda_{h}-\mu_{h}\left(\lambda_{l}+\lambda_{h}\right)}{\mu_{l} \lambda_{h}}+1\right]^{-1}
\end{gathered}
$$

Algorithm 2 Algorithm for Mobile Station (MS) Allocation Using Preemptive priority Model

MS (t) is the initial State of the MS at Time $t=0$ and Queue Size $<N$

If MCN Data Service request then

Allocate to MS for Service;

MS is Busy;

Else

If MS is Busy in Serving MCN Request AND Arriving MCN Request OR WSN Request then

Queue the Request;

$\mathrm{N}=\mathrm{N}+1$;

Else

Drop the Request;

End if

End if

If WSN Data Service request then

Allocate to MS for Service;

MS is Busy;

Else

If MS is Busy in Serving WSN Request AND Arriving MCN Request then

Preempt the WSN Service and Allow MCN in to Service;

Else

If MS is Busy in Serving WSN Request AND Arriving WSN Request then

Queue the Request;

$\mathrm{N}=\mathrm{N}+1$;

Else

End if

Drop the Request;

End if

End if

\subsection{Performance Indices (Preemptive Priority Model)}

The performance matrices required for QoS measurement has been calculated. The probability of arriving data packets from MCN and WSN is dropped due to busy mobile station and buffer space in queue is full is denoted as $\mathrm{P}_{\mathrm{D} \text { (preemptive) : }}$

$$
\mathrm{P}_{\mathrm{D} \text { (Preemptive) }}=\mathrm{P}_{(1,2)}+\mathrm{P}_{(2,1)}+\mathrm{P}_{(0,3)}+\mathrm{P}_{(3,0)}
$$


Probability that the mobile station is busy in serving low priority WSN data packets is:

$\mathrm{P}_{\mathrm{wsn}}=\mathrm{P}_{(1,0)}+\mathrm{P}_{(2,0)}+\mathrm{P}_{(3,0)}$

Probability that the mobile station is busy in serving at least one MCN data packet is:

$$
\mathrm{P}_{\mathrm{mcn}}=\mathrm{P}_{(0,1)}+\mathrm{P}_{(1,1)}+\mathrm{P}_{(2,1)}
$$

When the mobile device is busy in serving data packets, arriving MCN and WSN data packets has to wait in the queue if the queue is not full. The waiting time in queue is denoted by Wq (Preemptive) and can be calculated as:

$$
\mathrm{W}_{\mathrm{q}(\text { Preemptive })}=\mathrm{P}_{(1,2)}+\mathrm{P}_{(2,1)}+\mathrm{P}_{(1,1)}+\mathrm{P}_{(0,2)}+\mathrm{P}_{(2,0)}+\mathrm{P}_{(0,3)}+\mathrm{P}_{(3,0)}
$$

\section{NUMERICAL RESULTS}

In this section numerical analysis of both the models are presented. The QoS of the proposed models have been achieved by evaluating the performance parameters. The performance is evaluated in terms of dropping probability $\left(\mathrm{P}_{\mathrm{D}}\right)$, probability of high $(\mathrm{MCN})$ priority and low $(\mathrm{WSN})$ priority data in the system and waiting time $(\mathrm{Wq})$ of the data packets in the queue. The parameters taken for Figure 4 to Figure 11 are $\mu_{1}=1.0$; $\mu_{\mathrm{h}}=1.0 ; \lambda_{1}=0.5 ; \lambda_{\mathrm{h}}=0.1$ to 1.0 .

Figure 4 and Figure 5 represents the behavior of dropping probability $\mathrm{P}_{\mathrm{D}}$ with respect to increasing MCN data traffic load for both non-preemptive and preemptive cases respectively. It can be observed that with the increasing traffic load of MCN data, dropping probability increases for both the models. But by varying the traffic load of WSN the dropping probability decreases with decrease of WSN data traffic. Figure 4 shows a lower value of probability of dropping for non-preemptive model compared to preemptive model in Figure 5. This is due to the fact that data packets are served without preemption. Hence, by choosing proper data traffic load of WSN, dropping probability can be minimized to maintain QoS.

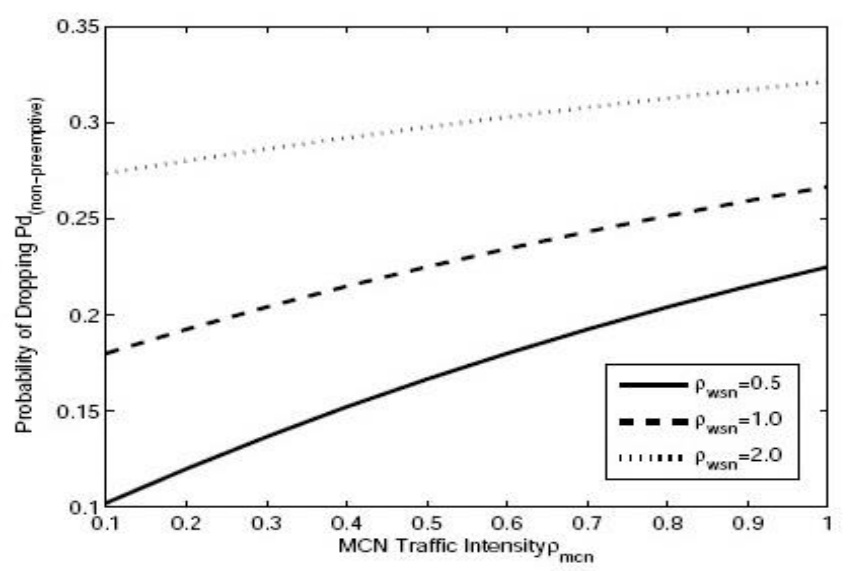

Figure 4. Traffic intensity of MCN Vs probability of dropping for non preemptive priority model

Figure 6 and Figure 7 depicts the probability of serving WSN data in the system for various WSN traffic load with respect to MCN traffic intensity in case of non preemptive and preemptive priority model respectively. It can be seen that with increase of MCN traffic load probability of serving WSN data decreases for both the models. This is due to continuous service of MCN data in non-preemptive priority model without preemption of WSN data where as WSN data preempted in preemptive priority model due to high priority MCN data. In Figure 7, preemptive priority model shows higher number of WSN data service in comparison with non preemptive priority model in Figure 6 which increases the WSN data service. Hence, preemptive priority model can achieve the better QoS for MCN and WSN by maintaining a balanced WSN traffic.

The probability of MCN data is in service increase with increase in MCN traffic intensity is shown in Figure 8 and Figure 9 for non-preemptive priority model and preemptive priority model respectively. It is observed that the probability of serving MCN data increases with increase in MCN traffic load for both the models. 


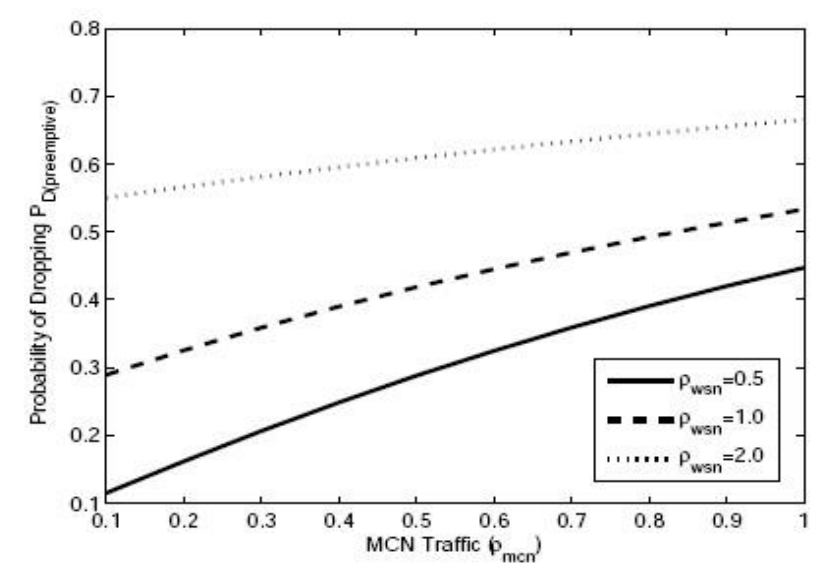

Figure 5. Traffic intensity of MCN Vs probability of dropping for preemptive priority model

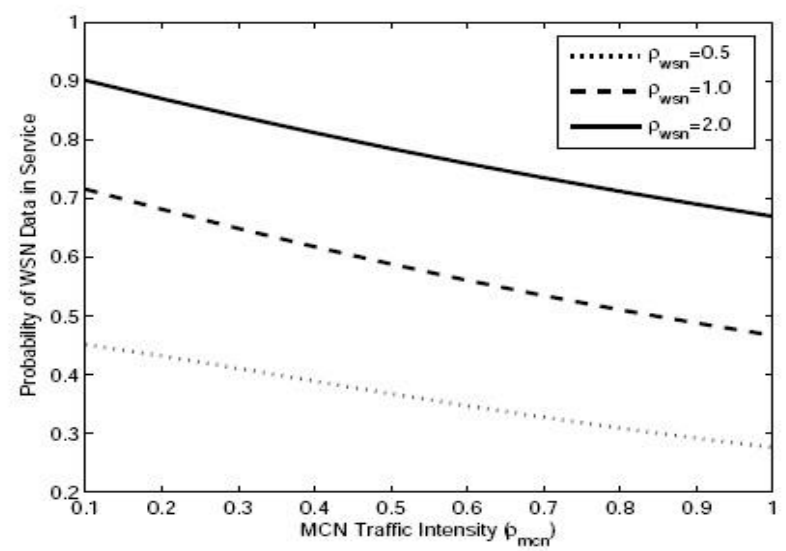

Figure 6. Traffic intensity of MCN Vs probability of WSN data in service for non preemptive priority model

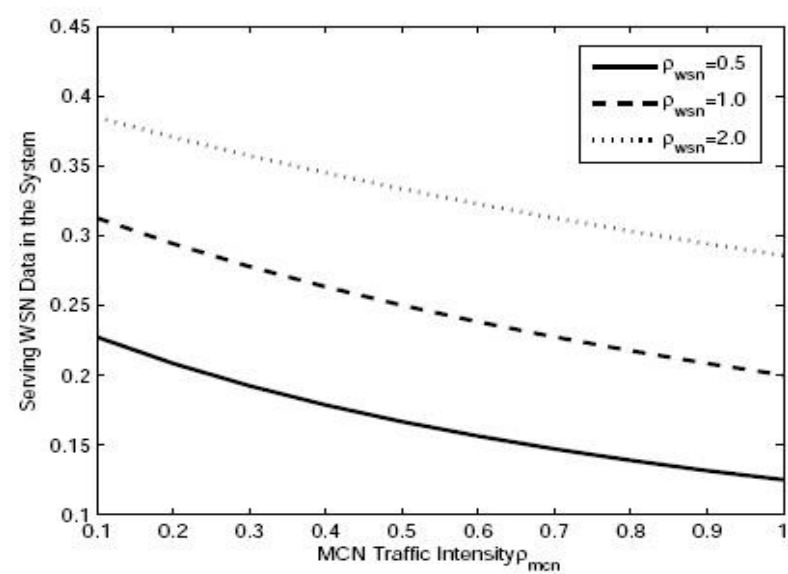

Figure 7. Traffic intensity of MCN Vs probability of WSN data in service for preemptive priority model

As MCN data have higher priority in preemptive priority model hence with admission of more number of MCN traffic, WSN data is preempted which increases the probability of serving MCN data as presented in Figure 8. In non-preemptive priority model probability of serving MCN data increases if MCN data admission 
increases in numbers as in Figure 9. But with increase of WSN data intensity, serving MCN data decreases in both models. Non preemptive priority model in Figure 8 shows little decrease in serving MCN data compared to serving MCN traffic in Figure 9 as WSN data are preempted from service in preemptive priority model which shows a better result.

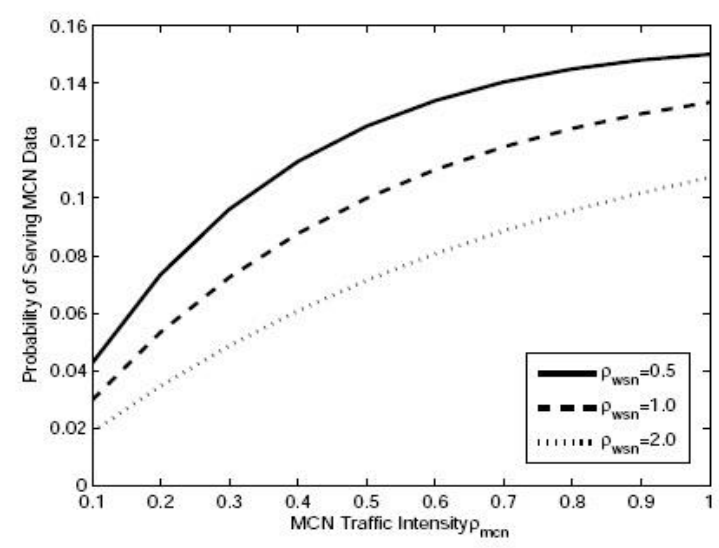

Figure 8. Traffic intensity of MCN Vs Probability of MCN data in service for non preemptive priority model

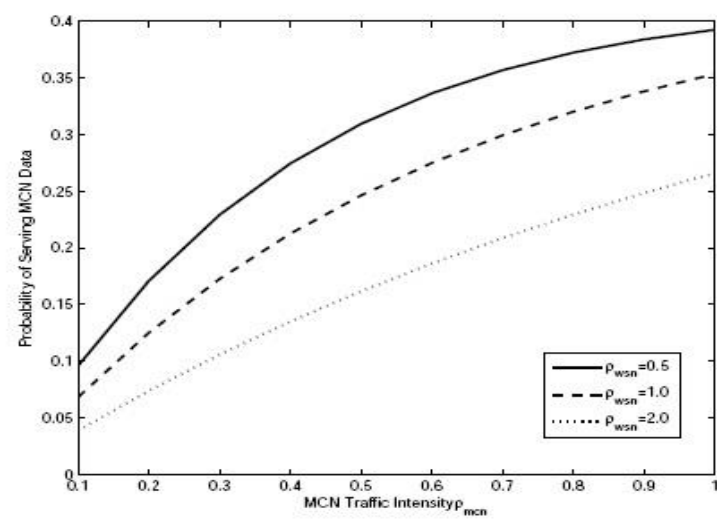

Figure 9. Traffic intensity of MCN Vs probability of MCN data in service for preemptive priority model

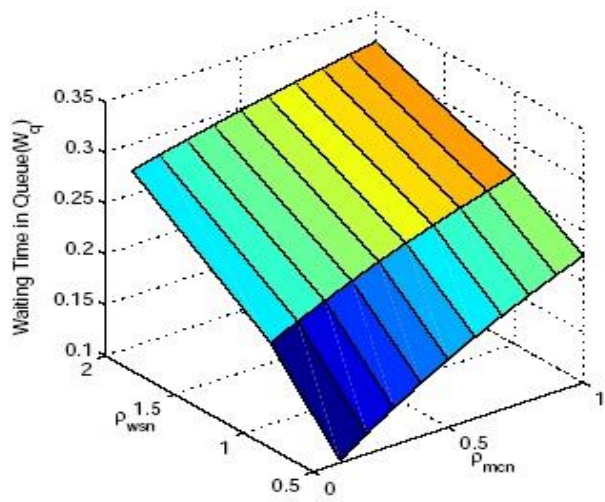

Figure 10. Traffic intensity of MCN, WSN Vs waiting time in queue for non preemptive priority model

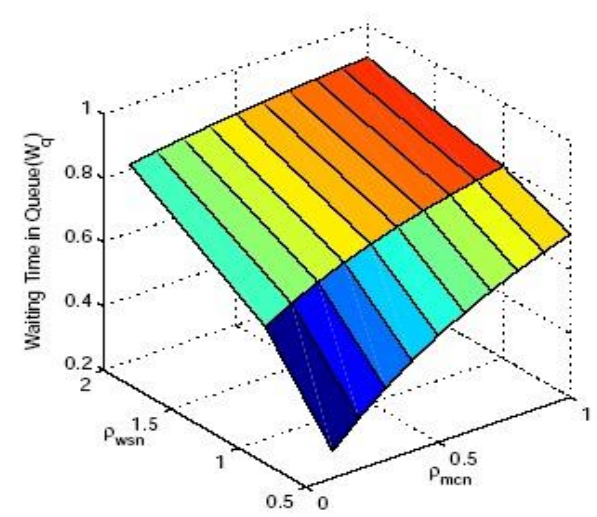

Figure 11. Traffic intensity of MCN, WSN Vs waiting time in queue for preemptive priority model 
Figure 10 and Figure 11 shows effect of traffic intensity $\rho$ on waiting time of data packets in queue for non preemptive priority model and preemptive priority models respectively. With increase in MCN data traffic the waiting time increases for both cases as MCN carries higher priority and preempt the existing WSN service shown in Figure 10. Also increase in WSN traffic waiting time increases for both the cases. This is due to arrival of MCN traffic which forces the WSN traffic to be in queue except the ongoing service for WSN data. But the waiting time in queue is found to be less for non-preemptive case compared to preemptive case. Hence, by regulating MCN traffic QoS can be maintained.

Figure 12 and Figure 13 shows a comparative analysis between non preemptive priority model and preemptive priority model for probability of serving of WSN and MCN data traffic with increase load of MCN traffic. In Figure 12 it can be observed that for preemptive priority model, probability of serving WSN data is higher compared to non-preemptive model. In Figure 13 preemptive priority model shows probability of serving MCN data traffic with higher value compared to non-preemptive priority model. Hence, by using preemptive priority model we can achieve QoS in WSN-MCN convergence data traffic.

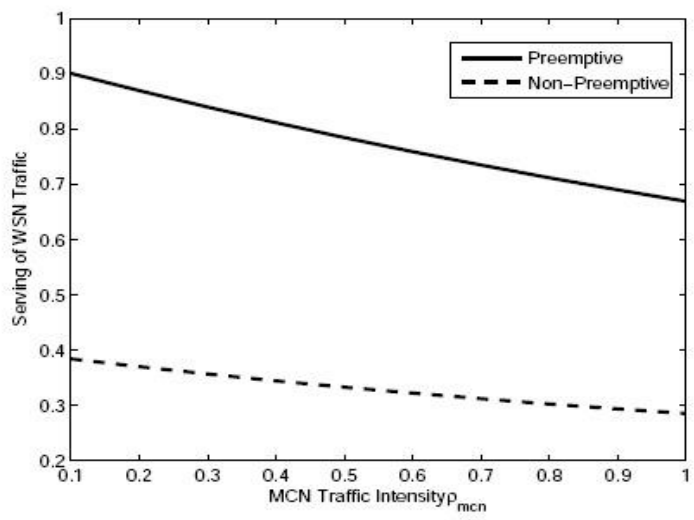

Figure 12. Traffic intensity of MCN Vs Probability of WSN data in service

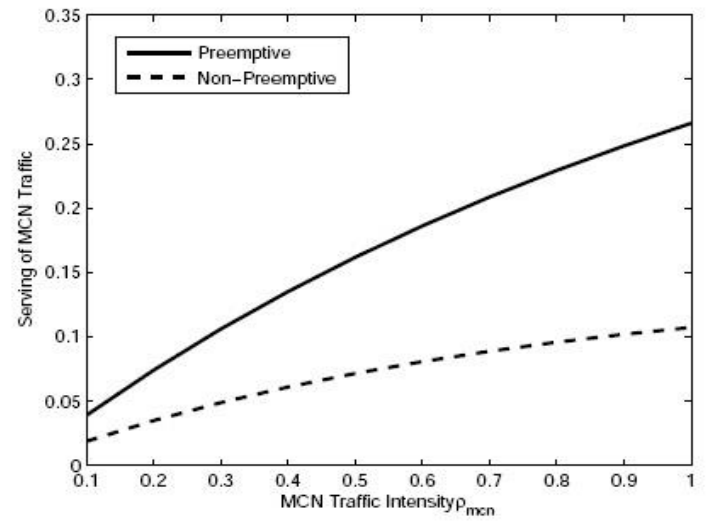

Figure 13. Traffic intensity of MCN Vs Probability of MCN Data in Service

\section{CONCLUSION}

We proposed two analytical models for preemptive and non preemptive based services at the MAC layer of WSN-MCN convergence network. As the MCN node act as the service point of both the type of network requests, hence a priority model is proposed to manage the network traffic. In the proposed schemes the performance indices in terms of blocking probability and dropping probability of data packets, waiting time in queue etc. has been determined for convergence network. A comparative numerical analysis is performed between both models and the preemptive model is proven to be efficient in handling both WSN and MCN requests. The QoS is achieved by maintaining a tradeoff between different performance metrics. 


\section{REFERENCES}

[1] Akyildiz IF, Su W, Sankarasubramaniam Y, Cayirci E. Wireless Sensor Networks: A Survey. Computer Networks. 2002; 38(4): 393-422.

[2] Verma PK, Verma R, Prakash A, Agrawal A, Naik K, Tripathi R, Alsabaan M, Khalifa T, Abdelkader T, Abogharaf A. Machine-to-Machine (M2M) communications: A survey. Journal of Network and Computer Applications. 2016; 66: 83-105.

[3] V. Karthikeyan. A Review on ZIGBEE in WSN. Indonesian Journal of Electrical Engineering and Computer Science. 2018; 9: 29-32.

[4] Munir SA, Ren B, Jiao W, Wang B, Xie D, Ma J. Mobile wireless sensor network: Architecture and enabling technologies for ubiquitous computing. In Advanced Information Networking and Applications Workshops, 2007, AINAW'07. 21st International Conference on 2007; 2: 113-120.

[5] Ekici E, Gu Y, Bozdag D. Mobility-based communication in wireless sensor networks. IEEE Communications Magazine. 2006; 44(7): 56-62.

[6] Zhang J, Shan L, Hu H, Yang Y. Mobile cellular networks and wireless sensor networks: toward convergence. IEEE Communications Magazine. 2012; 50(3): 164-169.

[7] Crosby GV, Vafa F. Wireless sensor networks and LTE-A network convergence. InLocal Computer Networks (LCN), 2013 IEEE 38th Conference on 2013; 731-734.

[8] Xiao J, Yin F, Wang H, Li Z, Liu F, Wang P. Energy-efficient data collection in WSN-MCN convergence architecture. In Communication Technology (ICCT), 2012 IEEE 14th International Conference on 2012; 524-530.

[9] Yin F, Li Z, Wang H. Energy-efficient data collection in multiple mobile gateways WSN-MCN convergence system. In Consumer Communications and Networking Conference (CCNC), 2013; 271-276.

[10] Yuan Z, Ouyang Y, Shan L, Hu H, Li Z. A Load Balancing Algorithm in Convergent Wireless Sensor and Cellular Networks. In Wireless Communications, Networking and Mobile Computing (WiCOM), 2012 8th International Conference on 2012; 1-6.

[11] Miao G, Azari A, Hwang T. E2-MAC: Energy efficient medium access for massive M2M communications. IEEE Transactions on communications. 2016; 64(11): 4720-35.

[12] Shan L, Li Z, Hu H. Converged Mobile Cellular Networks and Wireless Sensor Networks for Machine-to-Machine Communications. KSII Transactions on Internet \& Information Systems. 2012; 6(1): 147-161.

[13] Shan L, Fang W, Yao W, Xiong Y, Gao W. Adaptive Mobile Gateway: QoS-Guaranteed Challenges for Wireless Sensor Networks. InProceedings of the Second International Conference on Mechatronics and Automatic Control 2015; 1189-1195.

[14] Feng J, Zheng L, Fu J, Liu Z. An optimum gateway discovery and selection mechanism in WSN and mobile cellular network integration. In Communications and Networking in China (CHINACOM), 2013 8th International ICST Conference on 2013; 483-487.

[15] Xia J, Yun R, Yu K, Yin F, Wang H, Bu Z. Coordinated Multimode (Cellular-WSN) User Equipment Accessing Wireless Sensor Network Mechanism. InIntelligent Networking and Collaborative Systems (INCoS), 2012 4th International Conference on 2012; 550-555.

[16] Ouyang Y, Shan L, Yuan Z, Li Z. A cellular-assisted QoS-aware resource allocation algorithm for Wireless Sensor Networks. In Mobile Congress (GMC), 2011 Global 2011; 1-6.

[17] Ye W, Heidemann J, Estrin D. An energy-efficient MAC protocol for wireless sensor networks. In INFOCOM 2002. Twenty-First Annual Joint Conference of the IEEE Computer and Communications Societies. Proceedings. IEEE 2002; 3: 1567-1576.

[18] Panimozhi K, Mahadevan G. QoS Framework for a Multi-stack based Heterogeneous Wireless Sensor Network. International Journal of Electrical and Computer Engineering (IJECE). 2017; 7(5): 2713-2720.

[19] Mahboub A, Arioua M. Energy-efficient hybrid k-means algorithm for clustered wireless sensor networks. International Journal of Electrical and Computer Engineering (IJECE). 2017; 7(4): 2054-2060.

[20] Shan L, Fang W, Qiu Y, He W, Sun Y. Smart Mobile Gateway: Technical Challenges for Converged Wireless Sensor Networks and Mobile Cellular Networks. International Journal of Future Generation Communication and Networking. 2016; 9(9): 87-98.

[21] Gross D, Shortle JF, Thompson JM, Harris CM. Fundamentals of Queuing Theory. New York: John Wiley \&Sons. 2008. 